\title{
STARTOFF: WEB APPLICATION
}

\author{
Naman Dutta \\ SCOPE \\ VIT University \\ Vellore, India
}

\author{
Manish Paikara \\ SCOPE \\ VIT University \\ Vellore, India
}

\author{
Nandita Dutta \\ IMED \\ Bharati Vidyapeeth Deemed \\ University \\ Pune, India
}

\begin{abstract}
In the context of current scenario of eglobalization and free market economy, small scale businesses inhabit an essential place for employment, productivity and distribution of income. However, such businesses find themselves in an intensely competitive environment, absence of infrastructures, market analysis and financial difficulties. This paper identifies such problems of small-scale enterprises and provide answers for upgrading and modernizing their technologies through a platform. The platform will remove a wide range of barriers for acceptance of improved technologies. The platform is equipped with strong and feasible techniques for business analysis and growth. In our paper we are likewise exploring other aspects of strategy development like human resource, vendor development, association culture, investment etc.
\end{abstract}

Keywords - E-globalization, free market economy, Technology upgradation, Business analysis (key words)

\section{I.}

\section{INTRODUCTION}

As accessibility to e-commerce and technology has become a need for industries, large-scale industries are moving towards these markets. However small-scale industries are not able to compete with these industries as they do not have the capital or the technological initiate and run a successful business. This paper discusses about an ecommerce platform where small scale industries can trade their products with efficiency. The platform will also have an access market information, skills training materials and seminars for better understanding and improvement of their business. This platform also acts as a platform to showcase and improve their business. This allows investors to broaden their vision to small-scale industries and find small effective investments. Even the consumers will be able to get better products at cheaper rate as the small-scale industries generally have products which are locally sourced and cost less.

In 2020, many small-scale businesses and street vendors suffered as the Covid-19 pandemic raged all over the world. Such businesses were facing hardship in distribution of products, leading to business shutting down. Market all over the world had faced severe damage. Especially in India, where $45 \%-50 \%$ of exports are handled by small scale business, the damage dealt with the economy was immense. During this pandemic raged period, one of the many reasons why smallscale vendors were not able to continue and face a total shutdown was business not being conducted digitally.

\section{LITERATURE SURVEY}

In India, $50 \%$ of GDP is contributed by Small-scale industries. These industries do not have access towards modern technological enhancements. During the pandemic raged lockdown imposed small-scale vendors faced a total shutdown of the business. This showed us the need for introducing new technologies towards this sector of industry.[1] Currently, these small-scale industries are not able towards compete with large-scale industries, basically due towards the lack of capital. They lack the new technologies like e-commerce towards market their product towards the current market. They are facing constraints in competitive priorities, investment, , in addition performance optimization.[2] Despite the government-level knowledge that small-scale industries are not able towards cope with the e-commerce system of large industries, there has been no involvement towards uplift small-scale industries. The slow pace of e-commerce diffusion in small-scale industries has been studied, in addition found that investment, in addition marketing have been the furthermost important in the slow integration with e-commerce.[4] Currently, large eCommerce giants(large-scale industries) have utilized , in addition structured their marketing around social media. However, the small-scale industries do not have nor the capital nor the technological know-how for this level of marketing.[3] Many studies have shown that e-commerce is especially good for developing countries as it expands the market and availability to margin market.[5]

It is significant to note that recognition and acceptance for use of Information Technological tools are critical for further development of small-scale business. In general, acceptance can be defined as a positive choice towards utilize innovation. It is a common question why do businesses adopt new technologies. Answering this question will help many practitioners, in addition researchers towards improve, in addition improvise methods for designing, evaluating, , in addition planning the new technologies for firms. Through a small survey, we have analyzed that small-scale industries will adopt proposed application because of its usefulness and ease of use.

The paper has analyzed currently operating e-commerce services like Amazon and OLX for comparison with the platform it aims to create. Amazon takes $13-16 \%$ of sales, which generally less than the profit margin of small-scale industries.[16] Amazon also takes annual charges from the seller for using its platform which another problem for small industries. Amazon also has rules for inventory where certain 


\section{International Journal of Engineering Applied Sciences and Technology, 2021 \\ Vol. 6, Issue 2, ISSN No. 2455-2143, Pages 267-274 \\ Published Online June 2021 in IJEAST (http://www.ijeast.com)}

number of items has to be in the amazon warehouse from where all deliveries take place.[17]This increases cost to the seller. Comparing this to OLX provides only a platform and doesn't have any charge to the seller. However, it has no means of checking the product and has been a hot-spot for scammers. [18] This is especially prevalent in items which cannot be diagnosed easily. Both these platforms don't have any mechanism to check if seller is authorized under the rule of the government. This platform uses government identification as the main means for seller sign-up, thus linking the seller to a sue-able entity assuring product reliability. Instead of having warehouses, this platform allows for independent delivery services to deliver the products, which are chosen by the seller themselves. This decreases cost for both seller and the platform.

\begin{tabular}{|c|c|c|c|}
\hline Parameters & Amazon & OLX & $\begin{array}{l}\text { Proposed } \\
\text { Application }\end{array}$ \\
\hline $\begin{array}{l}\text { Product Manufacturing } \\
\text { Pressure }\end{array}$ & High & NA & Low \\
\hline Delivery Service & Centralized & Peer to peer & $\begin{array}{l}3^{\text {rd }} \text { Party }+ \\
\text { peer to peer }\end{array}$ \\
\hline Assured products & True & False & True \\
\hline Commodity Price & $\begin{array}{l}\text { As per } \\
\text { Market }\end{array}$ & $\begin{array}{l}\text { User's } \\
\text { Choice }\end{array}$ & $\begin{array}{l}\text { As per } \\
\text { Market }\end{array}$ \\
\hline Products Discounts & True & $\mathrm{NA}$ & True \\
\hline Merchant verification & False & False & True \\
\hline $\begin{array}{l}\text { Business Analysis } \\
\text { Service }\end{array}$ & False & False & True \\
\hline $\begin{array}{l}\text { Product } \\
\text { Recommendation }\end{array}$ & True & False & True \\
\hline $\begin{array}{l}\text { Mining frequent } \\
\text { itemset }\end{array}$ & True & False & True \\
\hline E learning Services & False & False & True \\
\hline Stock Market Analysis & False & False & True \\
\hline $\begin{array}{l}\text { Feedback \& Rating } \\
\text { Feature }\end{array}$ & True & NA & True \\
\hline
\end{tabular}

Fig 1: Gaps Identified in existing system and present system

III.

ANALYSIS

\section{A. Problems identified}

After examination of different constraints of small-scale industries, through field perceptions, oral interviews, , in addition questionnaires, data was gathered. This data was later processed using statistical techniques. Through this current, it's expected that restricted scale endeavors have really impacted the space of business and family wage. It also shows that there's a deficit of the bosses' abilities, vulnerable recordkeeping, in addition weak permission towards financing[6]. The serious complications faced by small scale industries remain:
- Deprived startup process[7]

- Lack of Infrastructure and resources impacts the ability of the small business towards enter export markets[8]

- Scarcity of dexterity, period, cash, , in addition sustenance keep up legitimate industrial procedures [9]

- Incapable to meet demands for various technological proficiencies [10]

- A lesser amount of susceptible towards tolerate a bombed endeavor for completing of ERP system[11].

- The excessive expense for business expansion projects [12]

- Unavailability of information between marketing, in addition production. [13]

- Shortage of skilled human resources towards operate small-scale businesses.[14]

\section{B. Solutions Proposed}

In the hour of e-globalization, bull bazaars have gotten amazingly novel, in addition savage. Earnestness has become advancement motivated. Toward invigorate their advancement, SSIs necessity to contribute their resources reasonably. Thus, it is typical that the power of the affiliations will depend upon interest cutting-edge diverse districts/growth. The followings are the courses of action stage will deal with sort:

- The platform can provide access to 3rd party services that will help small-scale businesses to tackle the problem of infrastructures, in addition resources.

- The platform is a web application, so the time of operations is done decisively.

- The platform will provide different advance assurance plans of the public authority, its offices just as bank crediting plans.

- The platform will try to meet the demands of several technological gaps, in addition help thrive small-scale businesses.

- The platform provides vague expertise in analyzing business growth, the need for Enterprise Resource Planning (ERP) system.

IV.

FRAMEWORK AND ARCHITECTURE

\section{A. MERN Stack framework}

The application is utilizing MERN stack towards fabricated the web application. It is a full-stack arrangement following the 3-level engineering: MongoDB as an information base, ReactJS as frontend, , in addition NodeJS as backend. 


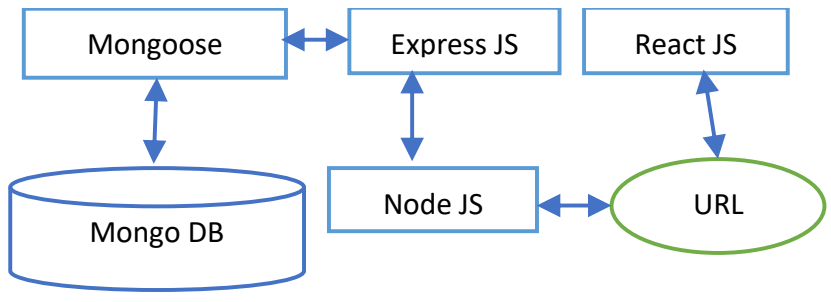

Fig 2: MERN stack Architecture

MongoDB is intended towards accumulate JSON information locally making it adaptable. It is based on JSON , in addition JavaScript. Express.js handles worker-side applications like HTTP solicitations, in addition reactions, likewise makes it simple towards plan URLs towards workerside capacities. React.js permits building intelligent UIs , in addition correspondence with far-off workers making it simple towards utilize, in addition convey.[15]

\section{B. Microservice Architecture}

The application has adopted microservices architecture Microservices - otherwise called the microservice architecture - is a building grace that erections a solicitation as an assortment of administrations that occur:

- Vastly workable, in addition demonstrable

- Sloppily attached

- Unconventionally galvanize

- Systematized round professional competencies

- Maintained via a minor squad

The micro-service architecture allows the quick, recurring and steadfast supply of huge, complicated applications. It similarly facilitates an association in the direction of progress its technology stack

$$
\text { V. }
$$

\section{SYSTEM REQUIREMENTS}

\section{A. Software Requirements}

Operating System : Windows 7/8/10, Linux, macOS

MongoDB: v3.6.6

Mongoose: v5.12.5

React Js: v17.0.1

Express: v4.17.1

Axios: v0.21.0

React-google-maps: v9.4.5

Node: v12.19.0

\section{B. Hardware Requirements}

CPU: $2 \mathrm{GHz}, 2$ core $\mathrm{CPU}$

GPU: GTX 710 (CUDA cores)

RAM: 6GB

Disk Space: $10 \mathrm{~GB}$

$$
\text { VI. }
$$

\section{PROPOSED SYSTEM}

This system uses user-based Collaborative filtering as the main recommendation algorithm. Collaborative filtering uses explicit rating of items as main factor for recommending products. This algorithm forms a product by user matrix of all the products and their rating, then decreases the matrix using PCA dimensionality reduction for easier computation and better average generation. Then similarity for all users with other's rating are used to predict values for non-rated items. This s then sorted and used for recommendation to each user. Aprori algorithm is also implemented to predict frequent item-sets from those bought by users.

For seller's business analysis is being done using time-series analysis. It is used in the platform to analyze sales, profit and stock data. It uses moving average, exponential smoothing and double exponential smoothing for better graphical representation. Sales data uses this graph to predict future sales. This transformed data can be utilized towards help create more effective strategies, in addition tactics for operational, in addition decision-making. Business Analysis conditions consist of mixture of improvements, submissions, measures, systems, products, in addition to dedicated models used towards empower the assortment, analysis, show, , in addition dissemination of interior, in addition exterior commercial facts.

\section{VII. \\ MODUlES AND DESCRIPTION}

\section{A. Product Recommendation Module}

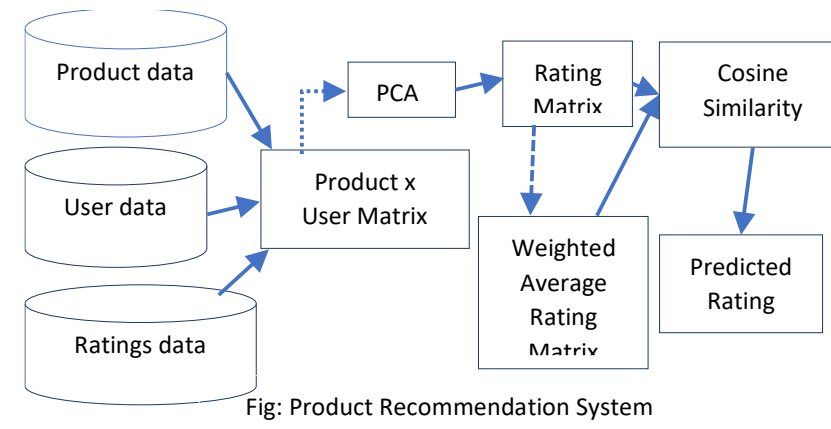

The recommender system makes prognostication based on users' historical practices. This service allows consumers towards get a recommendation for buying new products based on their previous data. The system will intake the rating of consumers, in addition find the similarity between users. Based on this similarity the application assumes that if user $\mathrm{A}$ has given good ratings towards product $\mathrm{X}$, in addition user $\mathrm{A}$, in addition user $\mathrm{B}$ have high similarity then user B might $\mathrm{b}$ interested in buying product $\mathrm{X}$.

After much analysis, the application decided that userbased Collaborative Filtering with explicit ratings of products would be a better approach towards the product recommendation service. After applying PCA (Principal Component Analysis) towards reduce dimension, in addition remove noise data, it has generated an $\mathrm{n} \times \mathrm{m}$ matrix of ratings, with user $u_{i}, i=1, \ldots n$, in addition product $p_{j}, j=1, \ldots m$. Now the application predicts the rating $\mathrm{r}_{\mathrm{ij}}$ if the target user I did not watch/rate an product $\mathrm{j}$ using equation 1 and in equation 2 . [20] 


$$
\begin{array}{r}
r_{i j}=\frac{\sum_{k}^{0} \text { Similarities }\left(u_{i}, u_{k}\right) r_{k j}}{\text { number of ratings }} \\
r_{i j}=\frac{\sum_{k}^{0} \text { Similarities }\left(u_{i}, u_{k}\right)\left(r_{k j}-\bar{r}_{k}\right)}{\text { number of ratings }}
\end{array}
$$

To calculate the similarity, the application can use Pearson's Correlation or Cosine Similarity. Since Pearson's Correlation works better with large data set, in addition the data set is small for now, the application is using Cosine Similarity towards find Similarities between users.

$$
\begin{gathered}
\text { Cosine Similarity }: \operatorname{Sim}\left(u_{i}, u_{k}\right)=\frac{r_{i} \cdot r_{k}}{\left|r_{i}\right|\left|r_{k}\right|}= \\
\frac{\sum_{j=1}^{m} r_{i j} r_{k j}}{\sqrt{\sum_{j=1}^{m} r^{2}{ }_{i j} \sum_{j=1}^{m} r^{2}{ }_{k j}}}
\end{gathered}
$$

To handle sparsity issues in the dataset, the application has created a temporary predicted rating. In this, wherever the user has not rated an product, the application has assigned a weighted mean average rating of that product in the place. the application has the user temporarily predicted ratings whenever the application encounters an product not rated. This helps towards handle the issue of calculating the similarity between users.

The Application is also using Apriori Algorithm towards recommend the user towards buy a product based on what products they have bought previously. The application is using products bought together dataset towards determine the frequent products set, in addition their support. For the system , the application has determined the length of frequent products set as 2 , in addition support value as a minimum of 4.

\section{B. Inventory Management Module}

Inventory management system can be tremendously valuable towards stock control, however, they shouldn't be mistaken for stock administration frameworks. Distribution center administration is tied in with engaging the stockroom group towards work as proficiently as could be expected while many stock frameworks may have some usefulness towards help with this, it will not be just about as exhaustive as a devoted warehousing apparatus[21].

Using the web service, the client can manage their entire inventory. They can register each different product, their combination for combination sales. This allows for easy overseeing of necessary purchases from sellers as well as sales towards customers. If the production of said product is done in-house it allows for better management of the production line. This Inventory system keeps multiple data related towards the product like the classification, tags, similar words, which makes it easier towards search for consumers, making it more user-friendly. The application can see a decrease in staff costs as separate manpower for inventory management is not required. It also decreases the chance of dead stock, in addition enhances transparency in the business.
The service is especially aimed towards the Clients(Seller). The seller can use the service towards manage, in addition control his entire inventory from the website. They can use manage sales, in addition purchases. The consumer can view the products sold by the clients. The consumer can book products from the inventory which can then be confirmed by the Seller. The administrator can view , in addition manage all Clients Inventory. This allows them towards solve any problems faced during running the services. They can also add products towards featured them, combine some of them for forming different sales campaigns.

\section{Shipping Management Module}

Shipping management is the function of applying processes towards guarantee the merchandise is adequately, in addition productively moved towards start with one area then onto the next. Now, in addition then called dispatch or armada the executives, it responds towards the inquiry. How would the application get this thing from direct A toward point B. As purchaser assumptions keep on rising, this capacity has never been in more prominent interest. In this article, the application will examine who is ordinarily responsible for conveyance the board inside an association , in addition how they can use programming towards do that work all the more adequately. Optimization that can be done are:

Routing Optimization: Rather than routing every single task by hand, course enhancement calculations can be used towards generate more productive courses in less time. Progressed enhancement programming can factor in limitations like conveyance windows, vehicle limits, driver plans, , in addition even traffic examples towards propose the ideal course for drivers. Course streamlining can likewise create assessed appearance times that can be utilized for inward, in addition client correspondence purposes.

Automated Dispatch: Maintaining an on-request conveyance business at scale requires mechanizing the dispatching interaction somewhat. In contrast towards planned conveyances, which can use forward-looking course streamlining, on-request organizations should have the option towards dispatch on the fly as new orders come in. Rather than attempting towards battle with an influx of approaching requests, connotations like Food Connect utilize robotized dispatching towards automatically dole out requests towards close drivers who can sensibly finish the request within the distributed period. Mechanizing dispatch opens up a conveyance director's valuable chance towards zero in on dealing with special cases, in addition guaranteeing clients are fulfilled.

Vehicle Tracking: Is a driver on their way towards their next conveyance or coincidentally heading the other way? Is it accurate towards say that they are trapped in rush hour traffic? Real-time tracking of vehicle locations can assist dispatchers with understanding when a driver might be offkilter, saving valuable assets, , in addition keeping away from exorbitant postponements. Vehicle following is additionally 


\section{International Journal of Engineering Applied Sciences and Technology, 2021 \\ Vol. 6, Issue 2, ISSN No. 2455-2143, Pages 267-274 \\ Published Online June 2021 in IJEAST (http://www.ijeast.com)}

utilized by client service groups towards advise clients when their conveyance will probably be finished.

The service itself does not provide any shipping services, however as the service is an open market system, this allows more competition, in addition also eases the shipping. For shipping companies' orders are provided with receiving point , in addition delivery point along with distance preferred, time. Having local delivery services will ease the last stretch delivery, which is the hardest towards manage.

\section{Online Payment Gateway}

Online Payment represents cash or cash like value transferred through electronic medium. It utilizes internet or intranet as a means of storing and transferring data required for transaction. Online payment ordinarily exchanges results of financial assets from two different banks or add record to your bank records. Currently online transcation accessible through Visa, Paypal, Google Pay, etc.

Traders using the web records need to conform to their safety rules and regulations. Online payment explicitly uses security as a means to diminish opportunity for third party to attain their individual records. The payment needs to occur over encrypted network connection to decreases such instances. Also these payment gateways should be intermittently checked for security flaws, so they can be solved in time, and data protected. As the service is completely online, there is also a need for an online payment gateway. As such the application is integrating Gateways like: ESEWA, KHALI, and CONNECTIONS. This will allow for easier management of the transaction. Especially connections as it is the only current service $n$ Nepal which allows for the easy inter-bank transaction. Payment from consumer towards client is notified towards the clients for easier transactions. Providing a wide variety of payment gateway eases the transaction flow for both consumers and clients.

\section{E. Business Analysis Module}

Business Forecasting Service is using time series analysis and autoregression forecasting. The application is extracting data of profits earned \& date of Clients, no of goods sold \& date of Clients and the weighted mean average of rating of sold products $\&$ date. With these data, the application is first smoothing the data (removing noise) in the period of 4 . Then the application is applying a chain of functions of Moving Average and Linear Weighted Moving Average with period 5 and period 2 respectively. Then after analyzing the data using sliding regression forecasting the application finds which forecasting is optimal Auto Regression using Least Square Method or Auto Regression using Max Entropy. The application also finds the sample size and degree for forecasting.

$$
S M A=\frac{A_{1}+A_{2}+\cdots+A_{n}}{n}
$$

$$
\operatorname{LWMA}=\frac{\left(\mathrm{P}_{\mathrm{n}} * \mathrm{~W}_{1}\right)+\left(\mathrm{P}_{\mathrm{n}-1} * \mathrm{~W}_{2}\right)+\ldots+\left(\mathrm{P}_{1} * \mathrm{~W}_{\mathrm{n}}\right)}{\sum \mathrm{W}}
$$

Also, the application can use cost-benefit analysis towards predict if the current business needs towards expand or not. The application is finding out the present value for future benefits and present value of future costs, from which we calculate Benefit Cost Ratio. This ratio will help determine whether the upgradation cost for the business in profitable or not.

Benefit Cost Ratio $=\frac{\sum \text { Present Value of Future Benef its }}{\sum \text { Present Value of Future Costs }}$

\section{F. E-Learning Module}

As the service is focused on small businesses, which work at the local level, they may not have the necessary skills in improving the quality of their product or how towards make the product cheaper. For this, the application is introducing E-learning services that allow them towards gain better knowledge in their trade and helps them towards improve their business. This in turn improves competition in the market and fosters innovation. Instead of completely building the E-learning service shorn of the mastery of different fields, the application is linking resources from multiple Learning services towards allow for better knowledge fostering. This is better for the business owner as they would be learning from industry professionals or experts in the field.

A learning framework dependent on formalized instructing yet with the assistance of electronic assets is known as E-learning. While educating can be situated in or out of the homerooms, the utilization of PCs and the Internet shapes the significant segment of E-learning. E-learning can likewise be named as an organization empowered exchange of abilities and information, and the conveyance of training is made towards countless beneficiaries at the equivalent or various occasions. Prior, it was not acknowledged wholeheartedly as it was expected that this framework did not have the human component needed in learning. E-learning has ended up being the best methods in the corporate area, particularly when preparing programs are directed by professionals for beginners across the globe and workers can get significant abilities while sitting in a boardroom, or by having courses, which are led for representatives of the equivalent or the various connotations under one rooftop. The schools which use E-learning advancements are a stride in front of those which have the conventional methodology towards learning.

\section{G. Authentication Module}

This service is using JWT security for authentication. JSON Web Token (JWT) is an open standard (RFC 7519) that defines a compact and self-contained way for securely transmitting information between parties as a JSON object. For the Reactjs JWT Authentication, the application used Backend Service (using Nodejs Express) provides secured RestAPIs with JWT token. - Reactjs project will request 


\section{International Journal of Engineering Applied Sciences and Technology, 2021 \\ Vol. 6, Issue 2, ISSN No. 2455-2143, Pages 267-274 \\ Published Online June 2021 in IJEAST (http://www.ijeast.com)}

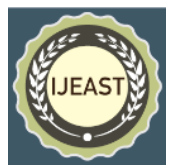

RestAPIs from the Backend system with the JWT Token Authentication implementation[19].

During the user registration phase, the user will use react JS form towards post essential data towards the backend. The backend code will verify the user details and finally accumulates the user signup information into the catalogue . During the user login phase, the user will post his/her either username or email along with the password towards backend code. After verifying if the username/email and password are correct, the Backend will create a secret JWT string and return it towards the user. After signing authentication is completed, the application extracts the JWT token from the Authorization Header in application local cache storage, with which the application sends a secured request towards the backend server of the application. For each request sent from the user, the backend code will scrutinize the JWT Authorization Header signature and then returns the required information data towards the user or user's registered authorities.

\section{H. Financial Module}

In this platform, the application has introduced an idea for loan processing. Various 3rd parties' fundraisers and common individuals can invest in these small-scale businesses by evaluating the business data of the vendors. Since these businesses are small, they have low-risk factors and no communal or political influences. Hence, these businesses are proper areas towards invest a small amount for individuals.

For any start-ups financing the project is the biggest problem. So towards help with this the application has a collection of different schemes and services provided by the administration and the banks. This can also be expanded towards include investors in the web service itself, which would allow start-ups towards pitch their ideas towards investors, this would allow them towards get better investment opportunities. The administration loans and programs take a significant part of the investment as it provides loan at significantly low interest, making it the best for start-ups.

The administration has set many such plans into action. Educated Youth Self-Employment Loan is provided towards youth with higher education towards start their own business. Youth below 40 years of age can get loans up towards 7 lakhs. For a subsidized interest rate of $5 \%$. This can be provided for entrepreneurs working in fields of agriculture, poultry, etc. The main criteria for the loan are towards hold a university degree, complete 7-day CTEVT training, and submit a brief proposal of the enterprise. Women Entrepreneurship Loan is provided towards uplift women. It has been specially provided towards improve women's condition as new entrepreneurs. They provide a loan of up towards 15 lakhs with an interest rate of $6 \%$. towards apply for this loan The system only needs towards provide a brief proposal of the enterprise. Similarly, loans and training are provided towards many sectors, towards promote entrepreneurship. They also have similar programs for commercial agriculture and the Dalit community. Recently administration has also started giving $50 \%$ of capital up towards 50 lakhs for technologyrelated startups at a $2 \%$ interest rate.

Similarly, administration also has many such initiatives, which it uses towards flourish startups. furthermost of these can be found at https://www.startupindia.gov.in. Some of those important towards small scale-business are; Dairy Entrepreneurship Development Scheme. Here farmers can get a $25 \%$ subsidy of the project cost and a contribution of $10 \%$ of the capital. Here farmers and NGOs can apply for this. Revamped Scheme of Fund for Regeneration of Traditional Industries is for artisans who want towards restart a traditional industry can apply for this. They can get up towards 8 crores if they have a cluster of at least 1000 artisans as hard intervention funding and 25lanks as soft intervention. These NGOs, institutions, and PRIs can apply for this.

Excluding these many other private funding s available in both countries. the service provides information about these. Forming a platform where entrepreneurs can easily get information. Later on, the application also plans towards attract banks and other similar financial institutions towards form an investment platform.

VIII.

USER CHARACTERISTICS AND FUNCTIONALITIES

\section{A. Characteristics}

1) Primary Stakeholders

- Consumer: They are the end-product buyers. Their User interface familiarity is unknown, so this should be made as easy towards use as possible. Technological knowledge is unknown, this has towards be taken care of while designing web service payment and order placement services.

- Client: They are the product manufacturers or local sellers. They have better knowledge of the user interface, as they are informed on how towards use the platform by administrators. They have at least enough knowledge towards run the interface, allowing a more complex user interface towards be placed for better control over the functions.

- Admin: They manage and maintain the website. They have good knowledge of the interface and underlying structure, allowing them towards make full use of the services. They have good knowledge of the website.

2) Secondary Stakeholders

- 3rd party Financial Service Provider

- 3rd party Learning Service Provider

- 3rd party Shipping Service Provider

- 3rd party Online Service Provider 


\section{International Journal of Engineering Applied Sciences and Technology, 2021 \\ Vol. 6, Issue 2, ISSN No. 2455-2143, Pages 267-274 \\ Published Online June 2021 in IJEAST (http://www.ijeast.com)}

\section{B. Functionalities}

- Consumer: Consumers register to the website by providing personal information. They can search, view details and order products. They can also add products to their wish-list. They can rate the product they have bought previously and provide feedback for the same. They can check the status of ordered product.

- Client: Client Registers towards the service by providing details about their business, including system registration. They can add, remove and update products from their inventory. They can add promocode to any of their products. They manage placed orders and deliver the product. They use Elearning platform for learning new skills and market information. They are provided with information of potential investors for their business.

- Admin: They verify if the client is genuine user or not. They can add, remove and edit products in client inventory. They can edit information about E-learning service. They also provides latest information about financial opportunities.

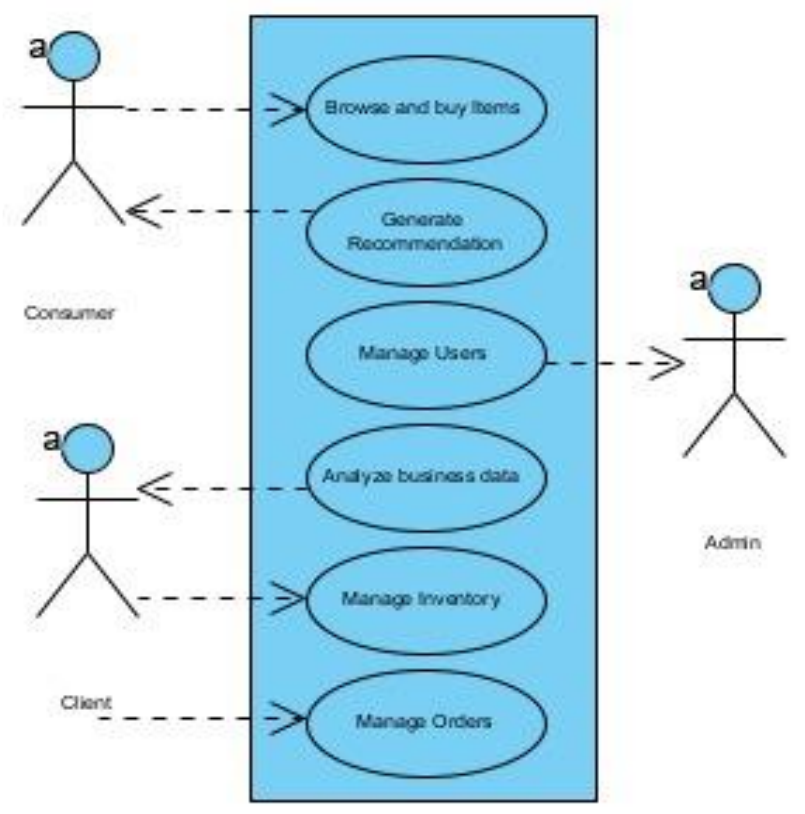

Fig: Use case Diagram

\section{CONCLUSION}

This paper demonstrates that e-commerce platform specifically for small-scale industries is required for them to compete in today's mass-production market. These smallscale industries will be able to provide specialized products for consumer problem compared to the generic solution provided by the large-scale industries. This platform allows small-scale industries to easily present their products to the consumers. In addition to selling capabilities, this platform also provides inventory management service which streamlines their procurement, manufacturing process and selling process. This help a business with capital to grow, however many entrepreneurs don't have any way to finance their business. For this the platform currently provides some information about government provided services. This is planned to improve into a crowdfunding platform, with information about sellers provided to allow them to better speculate about their growth. Many small-scale industries are stuck using old and inefficient manufacturing process, so to improve this the platform has an e-learning platform which currently provides minimal training videos in some fields. This can be improved to a platform where industry professionals can provide tutorials and give information about certification programs, conventions and training campaigns.

\section{REFERENCES}

[1] Sahu, Partha Pratim. Adoption of Improved Technology in India's Small-scale Industries: Evidences from a Field Survey. No. 0603. 2006.

[2] Narasimhan, Murali, Camelia Simoiu, and Anthony Ward. "Exposing commercial value in social networks: matching online communities and businesses." (2014).

[3] Narasimhan, Murali, Camelia Simoiu, and Anthony Ward. "Exposing commercial value in social networks: matching online communities and businesses." (2014).

[4] Macgregor, Robert, and Lejla Vrazalic. "The effect of small business clusters in prioritising barriers to Ecommerce adoption in regional SMEs." (2006): 24.

[5] Molla, Alemayehu, and Paul S. Licker. "eCommerce adoption in developing countries: a model and instrument." Information \& management 42.6 (2005): 877-899.

[6] Oboniye, Juliana A. "Small scale industries and rural development in Edo State, Nigeria." International Letters of Social and Humanistic Sciences 19 (2013): 158-169.

[7] Osamwonyi, Ifuero Osad, and Andrew E. Tafamel. "Options for sustaining small and medium scale enterprises in Nigeria: Emphasis on Edo state." African Research Review 4.3 (2010).

[8] Moen, Øystein. "The relationship between firm size, competitive advantages and export performance revisited." International Small Business Journal 18.1 (1999): 53-72.

[9] Gunasekaran, Angappa, et al. "Implications of organization and human behaviour on the implementation of CIM in SMEs: an empirical analysis." International Journal of Computer Integrated Manufacturing 14.2 (2001): 175-185.

[10] Narula, Rajneesh. "R\&D collaboration by SMEs: new opportunities and limitations in the face of globalisation." Technovation 24.2 (2004): 153-161.

[11] Muscatello, Joseph R., Michael H. Small, and Injazz J. Chen. "Implementing enterprise resource planning (ERP) systems in small and midsize manufacturing 
firms." International Journal of Operations \& Production Management (2003).

[12] March-Chorda, Isidre, A. Gunasekaran, and Begona Lloria-Aramburo. "Product development process in Spanish SMEs: an empirical research." Technovation 22.5 (2002): 301-312.

[13] Xiong, M. H., et al. "A DSS approach to managing customer enquiries for SMEs at the customer enquiry stage." International Journal of Production Economics 103.1 (2006): 332-346.

[14] Korea Federation of Small Businesses (2003), A Survey of Small Business on the Transfer of Production Facilities to Foreign Countries, Korea Federation of Small Businesses, Seoul (in Korean).

[15] Mai, Nhat. "E-commerce Application using MERN stack." (2020).
[16] Sell Amazon. https://sell.amazon.in/fees-andpricing.html

[17] Seller Central Amazon https://sellercentral.amazon.in/gp/help/external/G20033 $\underline{6920}$

[18] Repricer Express https://www.repricerexpress.com/ebay-vs-amazon/

[19] JWT

Authentication https://medium.com/@rempire230/user-authenticationwith-jwt-using-node-express-and-mongo-db-altasf0256232abb1

[20] Collaborative Filtering https://www.npmjs.com/package/collaborative-filter

[21] Stock Market data https://www.npmjs.com/package/alphavantage 